\title{
Lumbar Swelling as the Unusual Presentation of Henoch-Schonlein Purpura in a Child
}

\author{
Mehmet Ali Duman ${ }^{1}$, Nilgün Selçuk Durư ${ }^{1}$, Bahar Çalışkan ${ }^{1}$, Hale Sandıkç1 ${ }^{1}$, Ferhat Çengel $^{2}$
}

${ }^{1}$ Department of Pediatrics, Haseki Training and Research Hospital, İstanbul, Turkey

${ }^{2}$ Department of Radiology, Haseki Training and Research Hospital, İstanbul, Turkey

Background: Henoch-Schönlein Purpura (HSP) is a systemic hypersensitivity disease of unknown cause that is characterized by a purpuric rash and systemic manifestations, such as colicky abdominal pain, polyarthralgia, and acute glomerulonephritis. Common complications of HSP that lead to surgical intervention include intussusception, perforation, necrosis, and massive gastrointestinal bleeding. Unusual clinical manifestations of HSP may include edema of the scrotum and eyes. Lumbar swelling is rarely seen as a complication of HSP.

Case Report: A four-year-old boy was admitted to our emergency room with a 7 day history of increasing abdominal pain. Within 48 hours of admission, erythema- tous macules and palpable purpuric lesions developed on the lower extremities. On the third day of hospitalization, sudden onset of severe back pain, swelling on the lumbar region and refusal to walk were seen. On the fifth day, lumbosacral edema and pain resolved but facial edema developed. He was diagnosed clinically with HSP.

Conclusion: To the best of our knowledge, only a few cases of HSP with lumbar edema have been reported so far. If the first clinical symptoms of HSP are atypical, clinical progress can be atypical, too.

Keywords: Abdominal pain, Henoch Schonlein purpura, lumbar swelling, vasculitis, facial edema
Henoch-Schönlein purpura (HSP) is an acute, small-vessel vasculitis that most often affects the skin and internal organs. HSP is one of the most common vasculitis of childhood and it may develop at any age. The typical clinical characteristics are palpable purpura, abdominal pain and arthritis (1). Subcutaneous edema, which is a common finding in HSP, particularly appears in the hands, ankles, and feet $(2,3)$. Lumbosacral swelling is rarely seen in HSP, and to date, only a few cases have been reported in the literature (2). Here, we present a case of HSP with lumbar and facial edema.

\section{CASE PRESENTATION}

A four-year-old boy was admitted to our emergency room with a 7 day history of increasing abdominal pain. He was previously healthy without any remarkable past medical history.
On arrival to the emergency department, his vital signs were within normal limits. His blood pressure was 105/70 mmHg. Initial physical examination showed no abnormalities, except for abdominal pressing pain. No rash was noticed on the skin.

Laboratory assessment revealed a normal complete blood count, C-reactive protein, creatinine kinase, albumin, prothrombin time, and partial thromboplastin time. Analyses of serum biochemical test, Immunoglobulin and compliment levels were all within normal limits. Urine examination was also normal. Occult blood was positive in the feces. There were no pathologic findings in his abdominal ultrasound (Toshiba Aplio 500, Toshiba Medical Systems; Otawara, Japan).

Consultation of pediatric surgery revealed no abnormalities. Within 24 hours of admission, erythematous macules developed on the lower extremities. Later that evening, the erythematous macules on his lower extremities progressed to palpable purpuric lesions and spread to the buttocks. Biopsy from 
a purpuric lesion was performed. Histopathological findings were compatible with leukocytoclastic vasculitis. He was diagnosed as HSP and was treated with oral prednisolone with a dose of $1 \mathrm{mg} / \mathrm{kg} / \mathrm{day}$. On the third day of hospitalization, sudden onset of severe back pain, swelling on the lumbar region and refusal to walk were seen. A computed tomography (Brilliance 16, Philips Medical Systems; Cleveland, OH, USA) of the abdomen revealed the presence of thickening of intestinal loops (Figure 1), lumbosacral magnetic resonance imaging (Achieva, Philips Medical Systems; Best, The Netherlands) showed edema in the lumbar region. Oral prednisolone was increased to $2 \mathrm{mg} / \mathrm{kg} /$ day. On the fifth day, lumbosacral edema and pain resolved but facial edema developed (Figure 2). Ten days later, all symptoms of the patient resolved and occult blood was negative in the feces. He was discharged on steroid therapy which was continued for another 5 days. The monitoring of urine for hematuria and proteinuria was recommended, and the child presented a relapse only of the rash of the disease during the follow-up period for a month. Written and signed informed consent was obtained from the legal guardian for publication of this case report and the accompanying images.

\section{DISCUSSION}

Henoch-Schönlein purpura is an immune complex-mediated leukocytoclastic vasculitis with multi-system involvement. The annual incidence of HSP in children is about 20 per 100,000. The peak age of onset is between 4 and 6 years (1). The exact etiology of HSP is unknown; clinical evidence points to an infectious etiology. It is often preceded by an upper respiratory tract infection (1).

As there is no definitive test for diagnosis of the disease, it has been based on diagnostic criteria. Criteria were established by the American College of Rheumatology in 1990 and adopted by EULAR $\backslash$ PRINTO $\backslash$ PRES in 2008 (4,5). Our patient fulfilled four criteria (palpable purpura, young age, acute abdominal pain and leukocytoclastic vasculitis) of the American College of Rheumatology and three criteria (palpable purpura, abdominalpain, leukocytoclastic vasculitis) of EULAR PRINTO $\backslash$ PRES. He did not develop renal disease and arthritis or arthralgia. Unfortunately, immunofluorescence staining of the biopsy sample was not performed and histopathological examination was used for the diagnosis.

The predominant cutaneous finding of HSP is palpable purpura. Not only may maculopapular, urticarial, or vesicular lesions precede each other but they may also occur together with typical palpable purpura (1). Subcutaneous edema, which is a common finding in HSP, particularly appears in the hands, ankles, and feet while edema occurring in other locations is

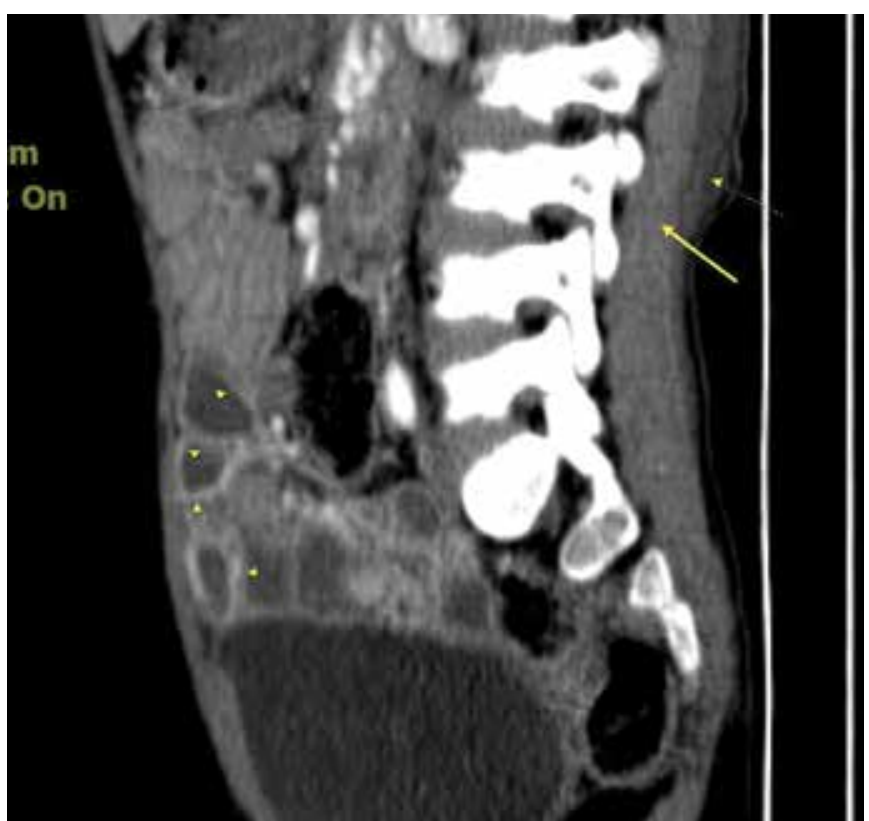

FIG. 1. Contrast-enhanced sagittal computed tomography image of the abdomen show hypodense appearance on lumbosacral subcutaneous tissue (dashed arrows) in comparison to the paraspinal muscles (arrows) due to edema. Also, increased thickness and contrast enhancement of the bowel wall (arrow heads) was thought to be secondary to vasculitis

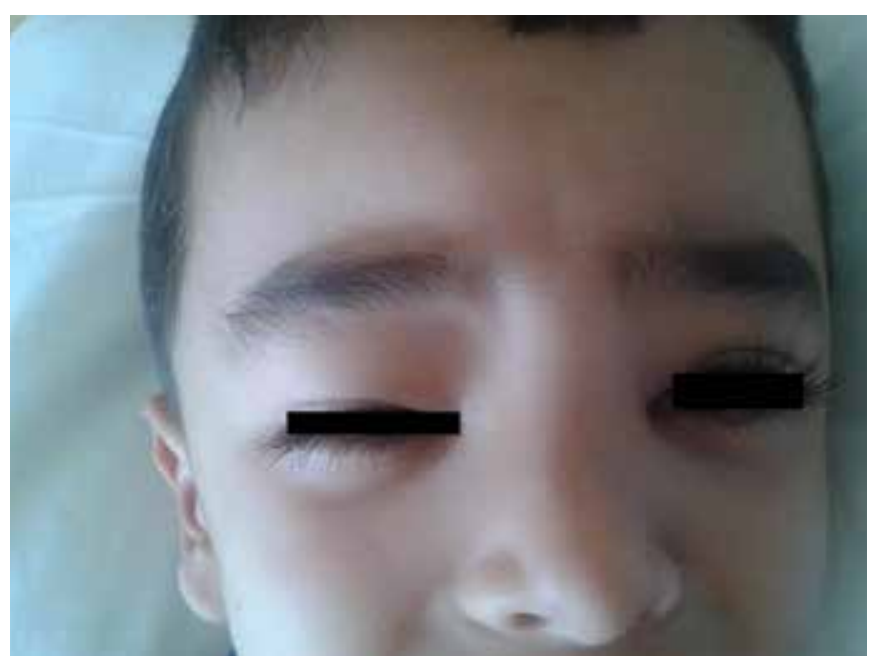

FIG. 2. Swelling of the periorbital area and forehead

uncommon $(2,3)$. Face, scalp, preno-scrotal and labial edema have been reported in a number of patients $(3,6-9)$. Facial edema is predominantly seen in children younger than 2 years (10). Schaefer et al. (2) reported the first case of lumbosacral edema as a presenting symptom of HSP in a 5-year-old boy. To the best of our knowledge, this is the second case of HSP with lumber edema reported in the literature. The back pain observed in our case was from the fluid accumulated in the soft tissue spaces, as it improved after resolving the edema. Unlike other cases in the literature, facial and lumbar edema 
were not the first clinical symptoms of HSP in our patient, who presented with abdominal pain and without a typical rash $(2,6)$.

In a previous study, the rate of HSP patients without skin rash in the first 24 hours of admittance has been shown to be nearly $5 \%$. They reported that these cases may be defined as atypical HSP (3). Our patient's first sign was abdominal pain without rash. In this case, erythematous macules which later turned into palpable purpura developed on the $8^{\text {th }}$ day after the onset of abdominal symptoms. The diagnosis of HSP is more challenging, especially when other symptoms precede skin lesions. The fact presented in this article is one of the extraordinary and rare cases. Therefore, the diagnosis process requires more attention and awareness.

Therapy for mild HSP cases is primarily supportive with analgesics and non-steroidal anti-inflammatory drugs. However, current literature shows that early use of corticosteroids in the treatment of HSP provide good clinical outcomes and prevent especially gastrointestinal co-morbidities (1).

Acute surgical pathologies and also HSP should be considered in differential diagnosis of the patients who presenting with acute abdominal pain. Facial and lumbar edema can be seen rarely in patients with HSP. Severe edema can cause severe pain and abnormal movement. If the first clinical symptoms of HSP are atypical, clinical progress can be atypical too.

\section{Ethics Committee Approval: N/A.}

Informed Consent: Written informed consent was obtained from the patient's parent who participated in this case.

Peer-review: Externally peer-reviewed.

Author contributions: Concept - M.A.D., N.S.D.; Design M.A.D., N.S.D.; Supervision - M.A.D., B.Ç., N.S.D.; Resource M.A.D., H.S., F.Ç., N.S.D.; Materials - M.A.D., H.S., N.S.D.; Data Collection and/or Processing - M.A.D., B.Ç., N.S.D.; Analysis and/ or Interpretation - M.A.D., B.Ç., N.S.D.; Literature Search - M.A.D., H.S., F.Ç.; Writing - M.A.D., B.Ç., N.S.D., F.Ç.; Critical Reviews B.Ç., N.S.D.
Conflict of Interest: No conflict of interest was declared by the authors.

Financial Disclosure: The authors declared that this study has received no financial support.

\section{REFERENCES}

1. Weiss PF. Pediatric vasculitis. Pediatr Clin North Am 2012;59:407-23. [CrossRef]

2. Schaefer B, Soprano C. Five-year-old boy presenting with severe back pain, swelling, and refusal to walk. Clin Pediatr (Phila) 2014;53:95-7. [CrossRef]

3. Anil M, Aksu N, Kara OD, Bal A, Anil AB, Yavaşcan O, et al. Henoch-Schönlein purpura in children from western Turkey: a retrospective analysis of 430 cases. Turk J Pediatr 2009;51:429-36.

4. Mills JA, Michel BA, Bloch DA, Calabrese LH, Hunder GG, Arend WP, et al. The American College of Rheumatology 1990 criteria for the classification of Henoch-Schönlein purpura. Arthritis Rheum 1990;33:1114-21. [CrossRef]

5. Ozen S, Pistorio A, Lusan SM, Bakkaloglu A, Herlin T, Brik $\mathrm{R}$, et al; Pediatric Rheumatology International Trials Organisation (PRINTO). EULAR/PRINTO/PRES criteria for HenochSchönlein purpura, childhood polyarteritis nodosa, childhood Wegener granulomatosis and childhood Takayasu arteritis: Ankara 2008. Part II: Final classification criteria. Ann Rheum Dis 2010;69:798-806. [CrossRef]

6. Hung TY, Liu MC, Hsu CF, Lin YC. Facial edema as the initial presentation of Henoch-Schonlein purpura in a 5-year-old boy. Pediatr Emerg Care 2009;25:31-2. [CrossRef]

7. Ceyhan M. Scalp and facial edema preceding Schönlein-Henoch syndrome by 28 days. Pediatr Dermatol 1992;9:311. [CrossRef]

8. Güneş M, Kaya C, Koca O, Keles MO, Karaman MI. Acute scrotum in Henoch-Schönlein purpura: fact or fiction? Turk $J$ Pediatr 2012;54:194-7.

9. Hiram-Karasmanis R, Smith RG, Radina M, Soboleski DA. Abdominal wall and labial edema presenting in a girl with Henoch-Schönlein purpura: a case report. J Med Case Rep 2010;4:98. [CrossRef]

10. Nussinovitch M, Prais D, Finkelstein Y, Varsano I. Cutaneous manifestations of Henoch-Schönlein purpura in young children. Pediatr Dermatol 1998;15:426-8. [CrossRef] 